\title{
EARLY COEFFICIENTS OF THE INVERSE OF A REGULAR CONVEX FUNCTION
}

\author{
RICHARD J. LIBERA AND ELIGIUSZ J. ZLOTKIEWICZ
}

\begin{abstract}
Using known properties of functions of positive real part it is shown that the first seven coefficients of the inverse of a regular univalent function mapping the disk onto a convex region are bounded by 1 .
\end{abstract}

1. Introduction. If $f(z)$ is in the class $S$, then

$$
f(z)=z+a_{2} z^{2}+a_{3} z^{3}+\ldots
$$

in the open unit disk $\Delta$ and $f(z)$ is one-to-one on $\Delta$. The inverse of $f(z)$ has a Maclaurin expansion in a disk of radius at least $\frac{1}{4}$, say

$$
\check{f}(w)=w+\gamma_{2} w^{2}+\gamma_{3} w^{3}+\ldots
$$

It has been conjectured [1] that the Koebe function $k(z)=z+\sum_{k=2}^{\infty} k z^{k}$ provides sharp bounds for all coefficients of $f(z),(1.1)$ and it has been shown that its inverse does provide the best bound for all $\left|\gamma_{k}\right|, k=2,3, \ldots$, over all members of $S$, [8]. Recently, Smale [9] made use of these ideas in developing a method for finding a zero of a complex polynomial.

Let $K$ be the subclass of $S$ containing all functions $f(z)$ for which $f[\Delta]$ is a convex region. It is known [6] that for $f(z)$ in $\mathcal{K},\left|a_{k}\right| \leq 1$ for all $k$ and that (except for a rotation of $\Delta)$ the function $T(z)=z /(1-z)=\sum_{k=1}^{\infty} z^{k}$ renders all these bounds sharp. Since $T(z)$ appears to have the same relation to $K$ as $k(z)$ does to $S$ it is reasonable to expect $T(z)$ to provide bounds for the inverses of functions in $K$. However,

$$
\check{T}(w)=\frac{w}{1+w}=w+\sum_{k=2}^{\infty}(-1)^{k+1} w^{k}
$$

converges in $\Delta$, whereas there are functions $f(z)$ in $K$ for which (1.2) converges only in disks of radius $\frac{1}{2}+\epsilon, \epsilon>0$; and for these, (1.2) cannot have bounded coefficients. These observations have been discussed elsewhere [3 and 4]. In an explicit demonstration Kirwan and Schober [3] show that for some $f(z)$ in $K,\left|\gamma_{n}\right|>$ 1 for $n \geq 10$. It is our purpose to examine bounds for $\left|\gamma_{n}\right|$ when $n$ is small.

THEOREM. For all $f(z)$ in $K,\left|\gamma_{n}\right| \leq 1, n=2,3,4,5,6,7$, and these bounds are sharp.

Our method of proof is classical and highly computational; in $\S 2$ we outline our methods and reproduce earlier and known results as lemmas and in $\S 3$ we outline the computations themselves.

Received by the editors March 7, 1981. Presented by title (Abstracts Amer. Math. Soc., 81T30-230, April 1981).

1980 Mathematics Subject Classification. Primary 30C50, 40C45; Secondary 30C50. 
2. Preliminary results. Using representations (1.1) and (1.2) together with $f(\breve{f}(w))=w$ or

$$
w=\check{f}(w)+a_{2}(\check{f}(w))^{2}+a_{3}(\check{f}(w))^{3}+\ldots,
$$

we obtain the relationships

$$
\left\{\begin{array}{l}
\gamma_{2}+a_{2}=0, \quad \gamma_{3}+2 a_{2} \gamma_{3}+a_{3}=0 \\
\gamma_{4}+a_{2}\left(\gamma_{2}^{2}+2 \gamma_{3}\right)+3 a_{3} \gamma_{2}+a_{4}=0 \\
\gamma_{5}+a_{2}\left(2 \gamma_{4}+2 \gamma_{2} \gamma_{3}\right)+a_{3}\left(3 \gamma_{3}+3 \gamma_{2}^{2}\right)+4 a_{4} \gamma_{2}+a_{5}=0 \\
\gamma_{6}+a_{2}\left(2 \gamma_{5}+2 \gamma_{2} \gamma_{4}+\gamma_{3}^{2}\right)+a_{3}\left(6 \gamma_{2} \gamma_{3}+3 \gamma_{4}+\gamma_{2}^{3}\right) \\
\quad+a_{4}\left(6 \gamma_{2}^{2}+4 \gamma_{3}\right)+5 a_{5} \gamma_{2}+a_{6}=0 \text { and } \\
\gamma_{7}+a_{2}\left(2 \gamma_{6}+2 \gamma_{2} \gamma_{5}+2 \gamma_{3} \gamma_{4}\right)+a_{3}\left(3 \gamma_{5}+6 \gamma_{2} \gamma_{4}+3 \gamma_{3}^{2}+3 \gamma_{2}^{2} \gamma_{3}\right) \\
\quad+a_{4}\left(4 \gamma_{4}+12 \gamma_{2} \gamma_{3}+4 \gamma_{2}^{3}\right)+a_{5}\left(5 \gamma_{3}+10 \gamma_{2}^{2}\right)+6 a_{6} \gamma_{2}+a_{7}=0
\end{array}\right.
$$

Let $P$ be the family of all functions $P(z)$ regular in $\Delta$ for which $\operatorname{Re}\{P(z)\}>0$ and

$$
P(z)=1+c_{1} z+c_{2} z^{2}+\ldots
$$

for $z$ in $\Delta$. Then we know that $f(z)$ is in $K$ if

$$
z f^{\prime \prime}(z)=f^{\prime}(z)[P(z)-1]
$$

for some $P(z)$ and $P$ and conversely. (2.4) is equivalent to the relations

$$
n(n-1) a_{n}=c_{n-1}+2 c_{n-2} a_{2}+\cdots+(n-1) c_{1} a_{n-1} .
$$

Successively eliminating $a_{k}$ 's on the right side of (2.5) yields

$$
\left\{\begin{array}{c}
2 a_{2}=c_{1}, \quad 6 a_{3}=c_{1}^{2}+c_{2}, \quad 24 a_{4}=2 c_{3}+3 c_{1} c_{2}+c_{1}^{3} \\
120 a_{5}=6 c_{4}+6 c_{1}^{2} c_{2}+8 c_{1} c_{3}+3 c_{2}^{2}+c_{1}^{4} \\
720 a_{6}=24 c_{5}+30 c_{1} c_{4}+20 c_{2} c_{3}+20 c_{1}^{2} c_{3} \\
+15 c_{1} c_{2}^{2}+10 c_{1}^{3} c_{2}+c_{1}^{5} \text { and } \\
(42)(120) a_{7}=120 c_{6}+144 c_{1} c_{5}+90 c_{1}^{2} c_{4}+90 c_{2} c_{4} \\
+40 c_{3}^{2}+120 c_{1} c_{2} c_{3}+40 c_{1}^{3} c_{3}+45 c_{1}^{2} c_{2}^{2} \\
+15 c_{2}^{3}+15 c_{1}^{4} c_{2}+c_{1}^{6}
\end{array}\right.
$$

Combining (2.2) and (2.6) makes it possible to express the $\gamma_{n}$ 's as polynomials in the $c_{k}$ 's, as is done in the next section. This then reduces the proof of Theorem 1 to a study of bounds on polynomials in the coefficients of functions in $P$. For this purpose we assemble the results which follow.

LEMMA 1. If $P(z)$ is in $P$ and as in (2.3), then $\left|c_{k}\right| \leq 2$ for each $k$.

This is a well-known result (see p. 41 of [6], for example).

LEMMA 2. If $P(z)$ is in $P$ and $s$ is a natural number, then

$$
\left|c_{n}-c_{n-s} c_{s}\right| \leq 2, \quad n \geq s, n=1,2,3, \ldots
$$

This result is due to A. E. Livingston [5]. 
LEMMA 3. For $P(z)$ in $P$, the expressions

$$
\left\{\begin{array}{c}
c_{2}^{*}=\left|c_{1}^{2}-c_{2}\right|, \quad c_{3}^{*}=\left|c_{1}^{3}-2 c_{1} c_{2}+c_{3}\right|, \\
c_{4}^{*}=\left|c_{1}^{4}+c_{2}^{2}+2 c_{1} c_{3}-3 c_{1}^{2} c_{2}-c_{4}\right|, \\
c_{5}^{*}=\left|c_{1}^{5}+3 c_{1} c_{2}^{2}+3 c_{1}^{2} c_{3}-4 c_{1}^{3} c_{2}-2 c_{1} c_{4}-2 c_{2} c_{3}+c_{5}\right|, \text { and } \\
c_{6}^{*}=\mid c_{1}^{6}+6 c_{1}^{2} c_{2}^{2}+4 c_{1}^{3} c_{3}+2 c_{1} c_{5}+2 c_{2} c_{4}+c_{3}^{2}-c_{2}^{3} \\
-5 c_{1}^{4} c_{2}-3 c_{1}^{2} c_{4}-6 c_{1} c_{2} c_{3}-c_{6} \mid
\end{array}\right.
$$

are all bounded by 2 .

The last lemma is obtained by an application of Lemma 1 to the reciprocal of $P(z)$ which is also in $P$. The function

$$
P_{0}(z)=1+\sum_{k=1}^{\infty} 2 z^{k}=\frac{1+z}{1-z}
$$

is one for which all inequalities in the above are sharp.

The last result we call upon is due to Carathéodory and appears in [2].

LEMMA 4. The power series for $P(z)$ given in (2.3) converges in $\Delta$ to a function in $P$ if and only if the Toeplitz determinants

$$
D_{n}=\left|\begin{array}{lllll}
2 & c_{1} & c_{2} & \cdots & c_{n} \\
c_{-1} & 2 & c_{1} & \cdots & c_{n-1} \\
\vdots & & & & \\
c_{-n} & c_{-n+1} & c_{-n+2} & \cdots & 2
\end{array}\right|, \quad n=1,2,3, \ldots,
$$

and $c_{-k}=\bar{c}_{k}$, are all nonnegative. They are strictly positive except for $P(z)=$ $\sum_{k=1}^{m} \rho_{k} P_{0}\left(e^{i t_{k}} z\right), \rho_{k}>0, t_{k}$ real and $t_{k} \neq t_{j}$ for $k \neq j$; in this case $D_{n}>0$ for $n<m-1$ and $D_{n}=0$ for $n \geq m$.

3. Proof of the theorem. Eliminating coefficients $a_{k}$ in (2.2) and (2.6) we obtain

$$
\left\{\begin{array}{c}
2 \gamma_{2}=-c_{1}, \quad 6 \gamma_{3}=2 c_{1}^{2}-c_{2}, \quad 24 \gamma_{4}=-6 c_{1}^{3}+7 c_{1} c_{2}-2 c_{3} \\
120 \gamma_{5}=24 c_{1}^{4}-46 c_{1}^{2} c_{2}+22 c_{1} c_{3}+7 c_{2}^{2}-6 c_{4} \\
720 \gamma_{6}=-120 c_{1}^{5}+96 c_{4} c_{1}+50 c_{2} c_{3}+326 c_{1}^{3} c_{2}-202 c_{1}^{2} c_{3} \\
-127 c_{1} c_{2}^{2}-24 c_{5} \text { and } \\
(120)(42) \gamma_{7}=-120 c_{6}+528 c_{1} c_{5}+1864 c_{1}^{3} c_{3}+1740 c_{1}^{2} c_{2}^{2} \\
+720 c_{1}^{6}+246 c_{2} c_{4}+100 c_{3}^{2}-1182 c_{1}^{2} c_{4} \\
-1292 c_{1} c_{2} c_{3}-5656 c_{1}^{4} c_{2}-127 c_{2}^{3} .
\end{array}\right.
$$

Using Lemmas 1 and 3 and the first four relations in (3.1) we have

$$
\begin{aligned}
2\left|\gamma_{2}\right| & \leq\left|c_{1}\right| \leq 2 \\
6\left|\gamma_{3}\right| & \leq\left|c_{1}\right|^{2}+\left|c_{1}^{2}-c_{2}\right| \leq 4+2=6 \\
24\left|\gamma_{4}\right| & \leq 2\left|c_{1}^{3}-2 c_{1} c_{2}+c_{3}\right|+\left|c_{1}\right|^{3}+3\left|c_{1}\right| \cdot\left|c_{1}^{2}-c_{2}\right| \\
& \leq 4+8+12=24
\end{aligned}
$$


and

$$
\begin{aligned}
120\left|\gamma_{5}\right| \leq & 6\left|c_{1}^{4}+c_{2}^{2}+2 c_{1} c_{3}-3 c_{1}^{2} c_{2}-c_{4}\right| \\
& +10\left|c_{1}\right| \cdot\left|c_{3}-2 c_{1} c_{2}+c_{1}^{3}\right| \\
& +8\left|c_{1}^{2}\right| \cdot\left|c_{1}^{2}-c_{2}\right|+\left|c_{2}\right|^{2} \\
\leq & 12+40+64+4=120 .
\end{aligned}
$$

These computations give correct bounds on $\left|\gamma_{k}\right|$ for $k=2,3,4,5$; we now make use of all four lemmas to obtain the proper bound on $\left|\gamma_{6}\right|$.

Again, from (3.1), we have

$$
\begin{aligned}
720\left|\gamma_{6}\right| \leq & 24\left|c_{5}+c_{1}^{5}+3 c_{1} c_{2}^{2}+3 c_{1}^{2} c_{3}-4 c_{1}^{3} c_{2}-2 c_{1} c_{4}-2 c_{2} c_{3}\right| \\
& +48\left|c_{1}\right| \cdot\left|c_{1}^{4}+c_{2}^{2}+2 c_{1} c_{3}-3 c_{1}^{2} c_{2}-c_{4}\right| \\
& +34\left|c_{1}\right|^{2} \cdot\left|c_{3}-2 c_{1} c_{2}+c_{1}^{3}\right| \\
& +2\left|c_{2}\right| \cdot\left|c_{3}-c_{1} c_{2}\right|+\left|14 c_{1}^{5}+5 c_{1} c_{2}^{2}-18 c_{1}^{3} c_{2}\right| .
\end{aligned}
$$

Using Livingston's Lemma (2, above) we see that $\left|c_{3}-c_{1} c_{2}\right| \leq 2$ and conclude that

$$
720\left|\gamma_{6}\right| \leq 520+2 \max \left|14 c_{1}^{4}+5 c_{2}^{2}-18 c_{1}^{2} c_{2}\right| \text {, }
$$

the maximum being taken over all admissible coefficients $c_{1}$ and $c_{2}$. We may assume without restriction that $c_{1}>0$, then from (2.10) we obtain

$$
D_{2}=\left|\begin{array}{lll}
2 & c_{1} & c_{2} \\
c_{1} & 2 & c_{1} \\
\bar{c}_{2} & c_{1} & c
\end{array}\right|=8+2 \operatorname{Re}\left\{c_{1}^{2} c_{2}\right\}-2\left|c_{2}\right|^{2}-4 c_{1}^{2} \geq 0
$$

from which we conclude that

$$
2 c_{2}=c_{1}^{2}+x\left(4-c_{1}^{2}\right)
$$

for some $x,|x| \leq 1$.

Now, using this representation for $c_{2}$ in the expression to be maximized in (3.3), we write

$$
\begin{aligned}
&\left|14 c_{1}^{4}+\frac{5}{4}\left[c_{1}^{4}+2 x\left(4-c_{1}^{2}\right) c_{1}^{2}+x^{2}\left(4-c_{1}^{2}\right)^{2}\right]-9 c_{1}^{4}-9 x c_{1}^{2}\left(4-c_{1}^{2}\right)\right| \\
&=\left|\frac{25}{4} c_{1}^{4}-\frac{13}{2} x c_{1}^{2}\left(4-c_{1}^{2}\right)+\left(4-c_{1}^{2}\right)^{2} x^{2}\right| \\
& \leq \frac{25}{4} c_{1}^{4}+\frac{13}{2}\left(4-c_{1}^{2}\right) c_{1}^{2}+\left(4-c_{1}^{2}\right)^{2} \\
&=\frac{3}{4} c_{1}^{4}+18 c_{1}^{2}+16 \leq 100 .
\end{aligned}
$$

(3.6) and (3.3) together show that $\left|\gamma_{6}\right| \leq 1$.

From the last relation in (3.1) it follows that

$$
\begin{aligned}
(120)(42)\left|\gamma_{7}\right| \leq & 120 c_{6}^{*}+288\left|c_{1}\right| \cdot c_{5}^{*}+246\left|c_{1}\right|^{2} c_{4}^{*} \\
& +28\left|c_{1}\right|^{3} c_{3}^{*}+10\left|c_{1}\right|^{4} c_{2}^{*}+20\left|c_{3}\right| \cdot\left|c_{1} c_{2}-c_{3}\right| \\
& +6\left|c_{2}\right| \cdot\left|c_{4}\right|+\left|28 c_{1}^{6}+64 c_{1} c_{2} c_{3}-90 c_{1}^{2} c_{2}^{2}-7 c_{2}^{3}\right| .
\end{aligned}
$$

All but the last term can be bounded correctly by bounds given in the first three lemmas above. Let

$$
A=\left|28 c_{1}^{6}+64 c_{1} c_{2} c_{3}-90 c_{1}^{2} c_{2}^{2}-7 c_{2}^{3}\right| .
$$

Using Carathéodory's criterion we will show that $A \leq 808$.

We may assume without restriction that $0 \leq c_{1} \leq 2$. Furthermore, if $0 \leq c_{1} \leq$ 1 , then it is easy to see that $A \leq 700$. 
Suppose, now, that $c_{1}=c$ and $1 \leq c \leq 2$, then some computation and proper grouping shows that $D_{3} \geq 0$, in (2.10), is equivalent to

$$
\begin{aligned}
\mid\left(4 c_{3}-4 c c_{2}+c^{3}\right) & \left(4-c^{2}\right)+c\left(2 c_{2}-c^{2}\right)^{2} \mid \\
\leq 2\left(4-c^{2}\right)^{2}-2\left|2 c_{2}-c^{2}\right|^{2} &
\end{aligned}
$$

Making use once again of (3.4) and (3.5) we rewrite (3.8) as

$$
4 c_{3}=c^{3}+2\left(4-c^{2}\right) c x-c\left(4-c^{2}\right) x^{2}+2\left(4-c^{2}\right)\left(1-|x|^{2}\right) z,
$$

for some $z$ with $|z| \leq 1$. Using (3.5) along with (3.9) we get

$$
\begin{aligned}
8 A= & \mid 101 c^{6}-184 c^{4}\left(4-c^{2}\right) x-c^{2}\left(4-c^{2}\right)\left(292-9 c^{2}\right) x^{2} \\
& -\left(4-c^{2}\right)^{2}\left(28+57 c^{2}\right) x^{3}+128 c\left(4-c^{2}\right)\left(1-|x|^{2}\right) \cdot\left(c^{2}+x\left(4-c^{2}\right)\right) z \mid
\end{aligned}
$$

and an application of the triangle inequality shows that

$$
\begin{aligned}
8 A \leq & {\left[101 c^{6}+128 c^{3}\left(4-c^{2}\right)\right] } \\
& +c\left(4-c^{2}\right)\left[512-128 c^{2}+189 c^{3}\right] \rho \\
& +c^{2}\left(4-c^{2}\right)\left[289-128 c-9 c^{2}\right] \rho^{2} \\
& +\left(4-c^{2}\right)\left[28-128 c+57 c^{2}\right] \rho^{3},
\end{aligned}
$$

with $\rho=|x| \leq 1$. Let $F(\rho)$ be the third degree polynomial on the right side of (3.10), then

$$
\begin{aligned}
F^{\prime}(\rho)=\left(4-c^{2}\right)\left\{512 c-128 c^{3}+189 c^{4}+\right. & 2\left[289 c^{2}-128 c^{3}-9 c^{4}\right] \rho \\
+ & \left.3\left(4-c^{2}\right)\left[28-128 c+57 c^{2}\right] \rho^{2}\right\} .
\end{aligned}
$$

$F^{\prime}(\rho)$ is a quadratic in $\rho$ with $F^{\prime}(0)>0, F^{\prime}(1)=\left(4-c^{2}\right)\left(336-1024 c+1178 c^{2}\right)>$ 0 and a negative coefficient for $\rho^{2}$, consequently $F$ is increasing and $\operatorname{Max}_{\rho} F(\rho)=$ $F(1)$. Now let

$$
G(c)=F(1)=448+1844 c^{2}+3 c^{4}-22 c^{6},
$$

then $G^{\prime}(c)=2 c\left(1844+6 c^{2}-66 c^{4}\right)>0$, so it follows that $G(c) \leq G(2)$. This shows that the upper bound for (3.10) corresponds to $\rho=1$ and $c=2$, in which case $8 A \leq(101)(64)$ and $A \leq 808$ for all admissible $c$. This concludes the proof of our theorem. All inequalities are rendered sharp by choosing $c_{k}=2, k=1,2,3, \ldots$.

4. Concluding remarks. Recently [7], Prokhorov and Szynal showed that $\left|\gamma_{n}\right| \leq$ $1, n=2,3,4$. For $n=2,3$ their method is essentially the same as ours; for $n=4$, however, they make use of Lemma 4, above, whereas our method relies solely on Lemmas 1 and 3. It appears, then, that our proof for $n=4$ is more elementary than theirs.

Kirwan and Schober [3] gave an upper bound for $\left|\gamma_{4}\right|$ and showed explicitly that $A_{10}=1.248 \ldots<$ for a given choice $\tilde{f}(w)$, i.e., $f(z)$ in $\mathcal{K}$. It is likely that $\left|\gamma_{n}\right| \leq 1$ for $n=8,9$.

The methods we have used above, particularly for $n=5,6,7$, require very delicate arrangement of the representations given in (3.1) so that the triangle inequality and the lemmas of $\S 2$ can be used. Applying the same techniques to $\gamma_{8}$ and $\gamma_{9}$ presents technical difficulties which may be too difficult to overcome: for in addition to the question of finding a suitable way of writing the representation in terms of coefficients $c_{k}$, one is faced with the necessity of making use of relations like $D_{n}>0, n=2,3,4,5$. 
ACKNOWLEDGEMENTS. Some of this work was done while the first author was either at FUB (Berlin) or UMCS (Lublin) under support of DAAD, NAS or PAN and concluded while the second author was a Visiting Professor at the University of Delaware.

\section{REFERENCES}

1. P. L. Duren, Coefficients of univalent functions, Bull. Amer. Math. Soc. 83 (1977), 891-911.

2. U. Grenander and G. Szegö, Toeplitz forms and their applications, Univ. of California Press, Berkeley and Los Angeles, 1958.

3. W. E. Kirwan and G. Schober, Inverse coefficients for functions of bounded boundary rotation, Tech. Rep. TR 79-42, MD 79-49-WK/GS, Univ. of Maryland, 1979.

4. J. G. Krzyż, R. J. Libera and E. J. Zlotkiewicz, Coefficients of inverses of regular starlike functions, Ann. Univ. Mariae Curie-Skłodowska Sect. A 33 (1979), 103-110.

5. A. E. Livingston, The coefficients of multivalent close-to-convex functions, Proc. Amer. Math. Soc. 21 (1969), 545-552.

6. Ch. Pommerenke, Univalent functions, Vandenhoeck and Ruprecht, Göttingen, 1975.

7. D. V. Prokhorov and J. Szynal, Inverse coefficients for $(\alpha, \beta)$-convex functions (preprint).

8. G. Schober, Coefficient estimates for inverses of schlicht functions (preprint).

9. S. Smale, The fundamental theorem of algebra and complexity theory, Bull. Amer. Math. Soc. 4 (1981), 1-36.

Department of Mathematical Sciences, University of Delaware, Newark, DELAWARE 19711

INSTytut Matematyki, UMCS, UL. NOWOtKi 10, 20-031 LUblin, POlaND 